\begin{tabular}{|l|l|l|}
\hline \multicolumn{2}{|c|}{ Publisherl nfo } \\
\hline PublisherName & $:$ & Springer International Publishing \\
\hline PublisherLocation & $:$ & Cham \\
\hline Publisherl mprintName & $:$ & Springer \\
\hline
\end{tabular}

\title{
The Inverse Problem for Elliptic Equations from Dirichlet to Neumann Map in Multiply Connected Domains
}

\section{Articlelnfo}

\begin{tabular}{|l|l|l|}
\hline ArticlelD & $:$ & 838 \\
\hline ArticleDOI & $:$ & $10.1155 / 2009 / 305291$ \\
\hline ArticleCitationID & $:$ & 305291 \\
\hline ArticleSequenceNumber & $:$ & 15 \\
\hline
\end{tabular}




\begin{tabular}{|c|c|c|}
\hline ArticleCategory & : & Research Article \\
\hline ArticleFirstPage & : & 1 \\
\hline ArticleLastPage & : & 1 \\
\hline ArticleHistory & : & $\begin{array}{ll}\text { RegistrationDate } & : \text { 2008-7-10 } \\
\text { Received } & : \text { 2008-7-10 } \\
\text { Accepted } & : 2009-1-5 \\
\text { OnlineDate } & : 2009-1-15\end{array}$ \\
\hline ArticleCopyright & : & $\begin{array}{l}\text { The Author(s) } 2009 \\
\text { This article is published under license to BioMed Central Ltd. } \\
\text { This is an open access article distributed under the Creative } \\
\text { Commons Attribution License, which permits unrestricted use, } \\
\text { distribution, and reproduction in any medium, provided the } \\
\text { original work is properly cited. }\end{array}$ \\
\hline ArticleGrants & & \\
\hline ArticleContext & : & 136612009200911 \\
\hline
\end{tabular}

Guochun Wen, Aff1

Corresponding Affiliation: Aff1

Email: wengc@math.pku.edu.cn

Zuoliang Xu, Aff2

Email: xuzl@ruc.edu.cn

Fengmin Yang, Aff2

Email: yangfengmin2006@126.com

Aff1 School of Mathematical Sciences, Peking University, Beijing 100871, China

Aff2 School of Information, Renmin University of China, Beijing 100872, China 


\section{Abstract}

The present paper deals with the inverse problem for linear elliptic equations of second order from Dirichlet to Neumann map in multiply connected domains. Firstly the formulation and the complex form of the problem for the equations are given, and then the existence and global uniqueness of solutions for the above problem are proved by the complex analytic method, where we absorb the advantage of the methods in previous works and give some improvement and development.

\section{Formulation of the Inverse Problem for Second-Order Elliptic Equations from Dirichlet to Neumann Map}

In [1-9], the authors posed and discussed the inverse problem of second-order elliptic equations. In this paper, by using the complex analytic method, the corresponding problem for linear elliptic complex equations of first-order in multiply connected domains is firstly discussed, afterwards the existence and global uniqueness of solutions of the inverse problem for the elliptic equations of second-order are obtained.

Let $G$ be an $N+1$-connected domain bounded domain in the complex plane $\mathrm{C}$ with the boundary $\partial G=L=U V_{j=0}{ }^{2} \in C_{\mu}^{2}(0<\mu<1)$, where $L_{j}(j=1, \ldots, N)$ are inside of $L$. Consider the linear elliptic equation of second-order:

$$
u_{z}+u_{\eta \eta}+3 u_{z}+b u_{\eta}=0 \text { in } G
$$

in which $\bar{a}=\vec{b}(\zeta), b=b(\zeta)$ are real functions of $\zeta=\xi+i n$, and $\left.\bar{\partial}(\zeta), b(\zeta) \in L_{D}(\bar{G}), b(\rangle 2\right)$ is a positive constant. Moreover let $\bar{a}=b=0$ in $\mathrm{C} \backslash G$. The above condition is called Condition $c$. In this paper the notations are the same as those in [10] or [11].

Denote 


$$
\begin{aligned}
& W(\zeta)=u+i b=\frac{\left[u_{\xi}-i u_{r}\right]}{2}=u_{\zeta} \\
& W_{\bar{\zeta}}=\frac{\left[W_{\xi}+i W_{r j}\right]}{2}=u_{\zeta \bar{\zeta}}=\frac{\left[u_{\xi \xi}+u_{r g}\right]}{4} \text { in } G \text {, }
\end{aligned}
$$

we can get

$$
\begin{aligned}
u_{\zeta \bar{\zeta}} & =W_{\bar{\zeta}} \\
& =\frac{1}{2}\left[W_{\xi}+i W_{\eta}\right] \\
& =-\frac{1}{4}\left[a u_{\zeta}+b u_{\eta}\right] \\
& =-\frac{1}{4}[a(W+W)+i b(W-\bar{W})] \\
& =-\frac{1}{4}[(a+i b) W+(a-i b) \bar{W}] \\
& =-A(\zeta) W-E(\zeta) \bar{W} \\
& =-2 \operatorname{Re}[A(\zeta) W] \text { in } G,
\end{aligned}
$$

where $A=A(\zeta)=\overline{B(\zeta)}=\bar{B}=[\bar{a}+i b] / 4$. We choose a conformal mapping $z=z(\zeta)$ from the above general domain $G$ onto the circular domain $D$ with the boundary $\Gamma=\cup{ }_{j}=0 j$, $\Gamma_{0}=\Gamma_{N+1}=\{|z|=1\}, \Gamma_{j}=\left\{|z-z|=,r_{j}\right\}, j=1, \ldots, N$, and $z=\square \in \Xi$. In this case, the complex equation (1.3) is reduced to the complex equation

$$
\begin{aligned}
& W_{\bar{z}}=-\overline{\zeta r(z)}\{A[\zeta(z)] W+B[\zeta(z)] \bar{W}\} \\
& w_{\bar{z}}=u_{z} \bar{z}=-2 \operatorname{Re}\left\{A[\zeta(z)] \overline{\zeta(z)} u_{z}\right\}=-2 \operatorname{Re}\left\{A[\zeta(z)] 7(z) w_{i}\right\} \text { in } D \text {, }
\end{aligned}
$$

where $u_{\zeta \bar{\zeta}}=u_{z} \bar{z}\left|z^{r}(\zeta)\right|^{2}, w(\zeta)=u_{\zeta}=u_{z^{2}} z^{\prime}(\zeta), w(z)=u_{z}, \zeta=\zeta(z)$ is the inverse function of $z=z(\zeta)$, and $\zeta^{\prime}(z)=1 / z^{r}(\zeta)=\overline{\gamma(z)}$ in $\bar{D}$ is a known Hölder continuously differentiable function (see [10, Section 2, Chapter I ]), hence the above requirement can be realized.

Introduce the Dirichlet boundary condition for (1.1) as follows:

$$
u=f(\zeta) \text { an } L=\partial G, \quad u=f[\zeta(Z)] \text { an } \Gamma=z(L)
$$


where $f(\zeta) \in C_{d}^{1}(L), f[\zeta(z)] \in C_{d}^{1}(\Gamma), a(\leq(D-2) / D)$ is a positive constant, which is called Problem o for (1.1) or (1.4). By [10, 11], Problem 0 has a unique solution $U^{U E W_{D}^{2}(G)}$ (or ${ }^{W}(D)$ ) satisfying (1.1) (or (1.4)) and the Dirichlet boundary condition (1.5). From this solution, we can define the Dirichlet to Neumann map $A: C_{a}^{1}(L) \rightarrow C_{a}(L)$ or $A: C_{a}^{1}(\Gamma) \rightarrow C_{a}(\Gamma)$ by $A f=\partial u / \partial n$.

Our inverse problem is to determine the coefficient $a$ and $b$ of (1.1) (or $A(\zeta)$ in (1.3)) from the map $A$. In the following, we will transform the Dirichlet to Neumann map $A$ into a equivalent boundary condition. In fact, if we find the derivative of positive tangent direction with respect to the unit arc length parameter $s=\arg z\left(z \in \Gamma_{0}\right)$ and $s=-\arg \left(z-z_{j}\right)\left(z \in \Gamma_{j}, j=1, \ldots, M\right)$ of the boundary $\Gamma$ with $s(0)=\arg z=\arg (1+0)=0$, then

$$
\begin{aligned}
f_{s} & =\frac{\partial f[\zeta(z)]}{\partial s} \\
& = \begin{cases}u_{z} z_{s}+u_{\bar{z}} \bar{z}_{s}=u_{z} i z-u_{\bar{z}} \bar{z}^{\bar{z}}=2 \operatorname{Re}\left[i z u_{z}\right], & \text { on } \Gamma_{0}, \\
u_{z}\left(z-z_{j}\right)_{s}+u_{\bar{z}} \overline{\left(z-z_{j}\right)} & \\
=-u_{z} i\left(z-z_{j}\right)+u_{\bar{z}} \overline{\left(z-z_{j}\right)} & \text { on } \Gamma_{j}, j=1, \ldots, N .\end{cases}
\end{aligned}
$$

It is clear that the equivalent boundary value problem is to find a solution $[W(\zeta(z)), u(\zeta(z))]$ of the complex equation (1.4) with the boundary conditions

$$
\operatorname{Re}[\overline{\lambda(z)} w(z)]= \begin{cases}\operatorname{Re}[i z w(z)]=\frac{f_{s}}{2}, & z \in \Gamma_{0}, \\ \operatorname{Re}[i(z-z), w(z)]=-\frac{f_{s}}{2}, & z \in \Gamma_{j}, j=1, \ldots, N, \\ u(1)=f[\zeta(1)]=b_{0},\end{cases}
$$

and the relation

$$
u(z)=2 \operatorname{Re} \int_{1}^{z} w(z) d z+b_{0} \text { in } \bar{D}
$$

in which $\lambda(z)=\bar{i}, z \in \Gamma_{0}$ and $\lambda(z)=\overline{i\left(z-z_{j}\right)}, z \in \Gamma_{j}, j=1, \ldots, N \cdot$ It is easy to see that 


$$
\begin{aligned}
2 \operatorname{Re} \int_{L_{j}} W(\zeta) d \zeta & =2 \operatorname{Re} \int_{\Gamma_{j}} u_{z} d z \\
& =-2 \operatorname{Re} \int_{0}^{s} i\left(z-z_{j}\right) u_{z^{r}}{ }_{j} d s \\
& =\int_{0}^{s_{j}} f_{s} r_{j} d s=0,
\end{aligned}
$$

where $\varsigma_{j}=2 \pi r j(j=1, \ldots, N)$ is the arc length of $\Gamma_{j}=\left\{\left|z-z_{j}\right|=r_{j}\right\}(j=1, \ldots, N)$ and applying the Green formula, we can see that the function $u(z)$ determined by the integral in (1.8) in $\bar{v}$ is single-valued.

Under the above condition, the corresponding Neumann boundary condition is

$$
\begin{aligned}
& u_{n}=\frac{\partial u}{\partial n}=u_{z} z_{n}+u_{\bar{z}} \bar{z}_{n} \\
& g(z)= \begin{cases}u_{z} z_{n}+u_{\bar{z}} \bar{z}_{n}=u_{z} z u_{\bar{z}} \bar{z}=2 \operatorname{Im}\left[i z u_{z}\right] & \text { an } \Gamma_{0}, \\
-u_{z}\left(z-z_{j}\right)-u_{\bar{z}} \overline{\left(z-z_{j}\right)}=-2 \operatorname{Im}\left[i\left(z-z_{j}\right) u_{z}\right] & \text { an } \Gamma_{j, j}, 1_{1} \ldots, N,\end{cases}
\end{aligned}
$$

where $n$ is the unit outwards normal vector of $\Gamma$. The boundary value problem (1.1) (or (1.4)), (1.10) will be called Problem $M$. Taking into account the partial indexes of $K_{0}=\Delta_{\Gamma_{0}}$ arg $[\lambda(z)]=\Delta_{\Gamma_{0}}$ arg $\overline{i z}$ and $\Delta \Gamma_{0}$ arg $\bar{z}$ are equal to -1 and $k_{j}=\Delta_{\Gamma_{j}} \arg \left[\lambda_{(z)]}=\Delta_{\Gamma_{j}} \arg \overline{\left(\overline{\left(z-z_{j}\right)}\right.}\right.$ and $\Delta_{\Gamma_{j}} \arg \overline{\left(z-z_{j}\right)}(j=1, \ldots, N)$ are equal to 1, thus the index of the above boundary value problem is $k=k_{0}+k_{1}+\cdots+k_{N}=N-1$. In general the above Problem $M$ is not solvable, we need to give the modified boundary conditions as follows:

$$
\begin{gathered}
\frac{1}{2} u_{n}=\operatorname{Re}\left[\overline{\lambda(z)} u_{z}\right]=\frac{g(z)}{2}+g_{0}, \quad z \in \Gamma_{j}, j=0,1, \ldots, N, \\
u(1)=b_{0} \quad \text { an } \Gamma,
\end{gathered}
$$

where $h(z)=\bar{z}, z \in \Gamma_{0}$, and $h(z)=\overline{z-z}, z \in \Gamma_{j}, j=1, \ldots, N, g(z) \in C_{d}(\Gamma)$ and $90=\square$ on $\Gamma_{j}(j=1, \ldots, N), 90$ on $\Gamma_{0}$ is an undetermined real constant (see [11, Chapter $\left.\mathrm{VI}\right]$ ). Hence, the Dirichlet to Neumann map can be transformed into the boundary conditions as follows: 


$$
\begin{aligned}
& u_{s}+i u_{n}= \begin{cases}2 \operatorname{Re}\left[i z u_{z}\right]+2 i \operatorname{Im}\left[i z u_{z}\right]=2 i z w(z), & z \in \Gamma_{0}, \\
-2 i(z-z) w(z), & z \in \Gamma_{j}, j=1, \ldots, N,\end{cases} \\
& w(z)=h(z)= \begin{cases}\frac{\left[u_{s}+i u_{n}\right]}{2 i z}, & z \in \Gamma_{0}, \\
-\frac{\left[u_{s}+i u_{n}\right]}{2 i(z-z)}, & z \in \Gamma_{j}, j=1, \ldots, N,\end{cases}
\end{aligned}
$$

which will be called Problem DN' for the complex equation (1.4) (or (1.1)) with the relation (1.8), where $h(z)\left(E C_{G}(\Gamma)\right.$ is a complex function satisfying the condition

$$
\int_{\Gamma_{j}} \operatorname{Re}[i(z-z j) h(z)] d s=0, \quad j=1, \ldots, N .
$$

For any function $f[\zeta(z)]\left(E C_{G}^{1}(\Gamma)\right)$ in the Dirichlet boundary condition (1.5), there is a set $\{g(z)\}$ of the functions of Neumann boundary condition (1.10), where $g(z)$ is corresponding to the complex equation (1.4) one by one, namely if we know the boundary value $f[\zeta(z)]$ and one complex equation in (1.4), then the boundary value $g(z)$ can be determined. Inversely if the $g(z)$ in (1.10) is given, then one complex equation in (1.4) can be determined, which will be verified later on. We denote the set of functions $\left\{e^{i k z_{h}}(z)\right\}$ by $R h$, where $k$ is a complex number and $h(z)$ is as stated in (1.12).

\section{Some Relations of I nverse Problem for Second-Order Elliptic Equations from Dirichlet to Neumann Map}

According to [10], introduce the notations

in which $f(z) \in L_{0}(D), p>2$. Suppose that $f(z)=0$ in $C \backslash D$. Obviously $(T f)_{z}=f(z)$ in $C$. We 
consider the complex equation

$$
\left.g \bar{z}+\jmath \mathrm{Ag}+e_{k}(z) B\right] \bar{g}=0, \quad g \bar{z}+\jmath \mathrm{Ag}+e_{k}(z) \overline{\mathrm{Ag}}=0 \text { in } \mathbf{C}
$$

where $g(z)=e^{i k z_{w i}}, e_{k}(z)=e^{i(k z+\overline{k z})}$ and $k$ is a complex number. On the basis of the Pompeiu formula (see [10, Chapters I and III]), the corresponding integral equation of the complex equation (2.2) is as follows:

$$
\left.g(z, k)-T\left[\exists A g+e_{k}\right] \overline{A g}\right]=\frac{1}{2 n i} \int_{\Gamma} \frac{g(\zeta, k)}{\zeta-z} d \zeta \text { in } D
$$

For simplicity we can only consider the following integral equation

$$
\left.g(z, k)-T\left[J A g+e_{k}\right] \overline{A g}\right]=1 \text { or } ; \quad \text { in } D
$$

later on.

Lemma 2.1.

If $f(z) \in L_{D}(D)(0>2)$, then

$$
\lim _{k \rightarrow \infty} \max _{z \in}\left|\left(T e_{k} f\right)(z)\right|=\square
$$

Proof.

It suffices to prove that for any small positive number $\varepsilon$, there exists a sufficiently large positive number $N$ such that

$$
|(T e, f)(z)|<\varepsilon \text { forze } \bar{D}, \mid k \varepsilon N
$$

In fact, noting that $e_{k}(z)=e^{2 i R e k z}=e^{2|k z| i \cos (\phi+a r g z)}, \phi=a r g k,\left|e_{k}(z)\right|=1$, and using the Hölder inequality, we have 
where $M_{1}=1+L_{p} f, 1<q=p /(D-1)<2$. Now we estimate the integral

We choose two sufficiently small positive constants $\bar{\delta}$ and $r$, and divide the domain $\sigma$ into three parts: $D_{1}=\{|\zeta| \leq \delta\}, D_{2}=\left\{D \backslash D_{1}\right\} \cap(\{|\operatorname{ar} g \zeta+\phi| \leq \eta\} \cup\{|\operatorname{ar} g \zeta+\phi-n| \leq n\})$, and $D_{3}=\bar{D} \backslash\left\{D_{1} \cup D_{2}\right\}$, such that for the above positive number $\varepsilon$, we can get

$$
\begin{aligned}
\left|z_{1}^{q}\right| & \\
& <\left(\frac{\varepsilon}{3 M_{1}}\right)^{q}, \\
\left|J_{1}\right| & <\frac{\varepsilon}{3 M_{1}}, \\
\left|J_{2}^{q}\right| & \\
& \leq\left(\frac{\varepsilon}{3 M_{1}}\right)^{q}, \\
\left|J_{2}\right| & \leq \frac{\varepsilon}{3 M_{1}},
\end{aligned}
$$

where $\theta=\arg \zeta$. Moreover noting that $|d(\theta+\phi)|=|d \cos (\theta+\phi) / \sin (\theta+\phi)| \leq \mid d \cos (\theta+\phi) / \sin \eta$, if $\zeta E D_{3}$, and then 


$$
\leq\left(\frac{\varepsilon}{3 M_{1}}\right)^{q}, \quad\left|J_{3}\right| \leq \frac{\varepsilon}{3 M_{1}} \text { for } \mid k E N
$$

Thus we obtain

$$
\begin{aligned}
\left|\left(T e_{k} f\right)(z)\right| & \leq\left|L_{p} f \jmath_{1}\right|+\left|L_{p} f z_{2}\right|+\left|L_{p} f z_{3}\right| \\
& \leq M_{1}\left(\left|z_{1}\right|+\left|j_{2}\right|+\left|\jmath_{3}\right|\right)<\varepsilon \text { for } z \in \bar{D},|k| \geq N .
\end{aligned}
$$

This shows that the formula (2.6) is true.

Lemma 2.2.

If $L_{D}[A, D] \leq k_{0}, D>2$, where $k_{0}$ is a positive constant, then the solution $g(z, k)$ of $(2.2)$ satisfies the estimate

$$
c_{a}[g(z, k), \bar{D}] \leq m_{2}=m_{2}\left(0, a, k_{0}, D\right)
$$

in which $M_{Z}$ is a positive constant.

Proof.

First of all, we verify that any solution $g(z, k)$ of $(2.2)$ satisfies the boundedness estimate

$$
C[g(z, k), \bar{D}] \leq m_{3}=m_{3}\left(0, a, k_{0}, D\right)
$$

where ${ }{ }_{3}$ is a positive constant. Suppose that (2.13) is not true, then there exists a sequence of coefficients $\left\{A_{m}(z)\right\}$, which satisfy the same condition of coefficient $A(z)$ and weakly converges to $A_{0}(z)$, and the corresponding integral equations 


$$
\left.9 m \bar{z}+7 A_{m G m}+e_{k}\right] \overline{A_{m} G_{m}}=0 \text { in } \bar{D}, m=1,2, \ldots
$$

possess the solutions $g_{m}(z, k)(m=1,2, \ldots)$, but $C\left[g_{m}(z, k), \bar{D}\right](m=1,2, \ldots)$ are unbounded. Hence we can choose a subsequence of $\left\{g_{m}(z, k)\right\}$ denoted by $\left\{g_{m}(z, k)\right\}$ again, such that $h_{m}=c\left[g_{m}(z, k), \bar{D}\right] \rightarrow \infty$ as $m \rightarrow \infty$, and can assume $h_{m} \geq 1$. Obviously $\tilde{g}_{m}(z, k)=g_{m}(z, k) / h_{m}(m=1,2, \ldots)$ are solutions of the integral equations

$$
\tilde{g}_{m \bar{z}}+\lambda A_{m} \tilde{q}_{m}+e_{k} \overline{A_{m} \tilde{q}_{m}}=0 \text { in } \bar{D}, m=1,2, \ldots .
$$

Noting that $L_{p}\left[A_{m} \tilde{G}_{m}\right] \leq k_{0}, L_{p}\left[e_{k}, \overline{A_{m} \tilde{G}_{m}}, \bar{D}\right] \leq k_{0}$, we can derive the estimate

$$
\left.\left.C_{a}[T] A_{m} \tilde{g}_{m}+T e_{k}\right] \overline{A_{m} \tilde{q}_{m}}, \bar{D}\right] \leq m_{4}=m_{4}\left(0, a, k_{0}, D\right)
$$

(see $[10,11])$, thus

$$
C_{a}\left[\tilde{G}_{m}, \bar{D}\right] \leq M_{5}=M_{5}\left(0, a, k_{0}, D\right) .
$$

Hence from $\left\{\tilde{G}_{m}(z, k)\right\}$, we can choose a subsequence denoted by $\left\{\tilde{G}_{m}(z, k)\right\}$ again, which uniformly converges to $\widetilde{q}_{0}(z)$ in $\bar{D}$, it is clear that $\widetilde{q}_{0}(z)$ is a solution of the equation

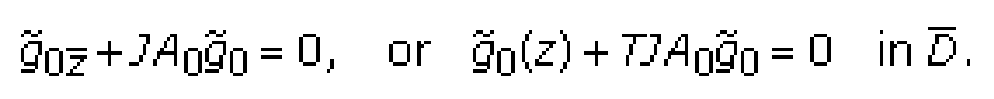

On the basis of the result in $\left[10\right.$, Section 5 , Chapter III], the solution $\widetilde{g}_{0}(z)=\square$ in $\bar{D}$, however, from $C\left[\tilde{g}_{m}(z, k), \bar{D}\right]=1$, there exists a point $z^{*} \in \bar{D}$, such that $C\left[\tilde{g}_{0}\left(z^{*}\right), \bar{D}\right]=1$, which is impossible. This shows that (2.13) and then the estimate (2.12) are true.

Lemma 2.3.

Under the above conditions, one has

$$
\lim _{k \rightarrow \infty} g(z, k)=g_{0}(z) \text { in } \bar{D}
$$


where $90(z)$ is a unique solution of the equation

$$
90 \bar{z}+7 A g_{0}=\square \text { in } \bar{D}
$$

Proof.

Denote by $g(z, k)$ the solution of (2.2) in $\bar{D}$. From Lemma 2.2, we know that the solution $g(z, k)$ satisfies the estimate (2.12). Moreover by using (2.5), that is,

$$
\lim _{k \rightarrow \infty} \max _{z \in \bar{D}}\left|\left(T e_{k} \overline{A g}\right)(z)\right|=0
$$

we can choose subsequences $\left\{k_{n}\right\}$ and $\left\{g\left(z, k_{n}\right)\right\}$, where $k_{n} \rightarrow \infty$ as $n \rightarrow \infty$, such that $\left\{g\left(z, k_{n}\right)\right\}$ in $\bar{b}$ uniformly converges to $g_{0}(z)$ as $n \rightarrow \infty$, which is a solution of (2.20) in $\bar{v}$ (see [11]). The uniqueness of solutions of (2.20) can be seen from the proof of Lemma 2.4 below.

Lemma 2.4.

The solution $90(z)$ of $(2.20)$ can be expressed as

$$
90(z)=\Phi(z) e^{-T J A} \text { in } \bar{D}
$$

where $\Phi(z)=1$ in 0 .

Proof.

On the basis of the results as in [10, Section 5, Chapter III], we know that the integral equations

$$
g_{0}(z)-7>A g_{0}=\left\{\begin{array}{l}
1 \text { in } \bar{D} \\
1 \text { in } \mathrm{C}
\end{array}\right.
$$

have the unique solutions $9_{0}(z)$ in $\bar{D}$ and $\mathrm{C}$ respectively, this shows that the function $9_{0}(z)$ in $\bar{D}$ can be extended in $\mathrm{C}$. Moreover by the result in $[10,11]$, the solution $9_{0}(z)$ can be expressed 
as $W(z)=g_{0}(z)=\Phi(z) e^{-T J A}$ in $\mathrm{C}$. Note that $\tau z A \rightarrow \square$ as $z \rightarrow \infty$, and the entire function $\Phi(z)$ in $\mathrm{C}$ satisfies the condition $\Phi(z) \rightarrow 1$ as $z \rightarrow \infty$, hence $\Phi(z)=1$ in $C$, and then $g_{0}(z)=e^{-T J A}$ in $\bar{D}$.

\section{Theorem 2.5.}

For the inverse problem of the equation

$$
\left[g_{0}(z)\right]_{\bar{z}}+3 A_{Q_{0}}=0 \text { in } \bar{D}
$$

with the boundary condition

$$
g_{0}(z)(\neq \equiv) \text { on } \Gamma \text {, }
$$

one can obtain

$$
\nabla J A=-\ln g_{0}(z) \text { on } \Gamma
$$

which is a known function.

Proof.

From the expression (2.22) of the solution $g_{0}(z)$ in $\bar{D}$ and $\Phi(z)=1$ in $\bar{D}$, it follows that (2.26) is true.

\section{The Inverse Scattering Method for Second-Order Elliptic Equations from Dirichlet to Neumann Map}

For the complex equation (1.4), through the transformation $W(z)=w(z) e^{T J A}$, we can obtain that the function $W(z)$ satisfies the complex equation 


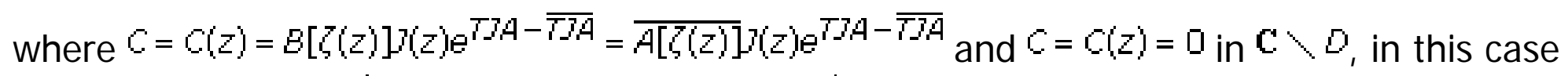
every function $h(z) e^{i k z}$ in $R_{A}$ is reduced to $h(z) e^{i k z+T z A}$, hence later on it suffices to discuss the complex equation (3.1) and system of complex equations

$$
\varphi_{j \bar{z}}+(-1)^{j-1} \mathrm{C}(z) e_{k}(z) \overline{\varphi_{j}}=0 \text { in } \mathbf{C}, j=1,2 \text {. }
$$

where $e_{k}(z)=e^{i(k z+\overline{k z})}$. In the following we will find two solutions $\phi_{1}(z)$ and ${ }^{i \phi_{2}(z)}$ of complex equation $[\phi]_{\bar{z}}+C(z) e_{k}(z) \overline{\phi(z)}=0$ with the conditions $\phi_{1}(z) \rightarrow 1$ and $i \phi_{z}(z) \rightarrow i$ as $z \rightarrow \infty$.

Now we find two solutions $W_{1}(z)$ and $W_{2}(z)$ in $C$ of (3.1) with the conditions $W_{1}(z) \cdots e^{-i k z}$ and $W_{2}(z) \cdots i e^{-i k z}$ for sufficiently large $|z|$. In other words, there exist two solutions $\phi_{1}(z)=e^{i k z} W_{1}(z)$ and $\phi_{2}(z)=-i e^{i k z} W_{2}(z)$ in $C$ of $(3.2)$ with the conditions $\phi_{1}(z) \rightarrow 1$ and $\phi_{2}(z) \rightarrow 1$ as $z \rightarrow \infty$. Denote

$$
m_{1}(z, k)=\frac{\left[\phi_{1}(z)+\phi_{2}(z)\right]}{2}, \quad m_{2}(z, k)=e_{k}(z) \frac{\left[\overline{\varphi_{2}(z)}-\overline{\varphi_{1}(z)}\right]}{2},
$$

obviously $m_{1}(z, k), m_{2}(z, k)$ satisfy the system of first-order complex equations

$$
\begin{gathered}
{\left[m_{1}\right]_{\bar{z}}=c m_{2}, \quad\left[m_{2}\right]_{z}-i k m_{2}=\bar{c} m_{1}, \quad e_{k}\left[e_{-k} m_{2}\right]_{z}=\bar{c} m_{1}} \\
{\left[m_{2}\right]_{z}=e_{k}\left[\frac{\overline{q_{2}}-\overline{m_{1}}}{2}\right]_{z}+i k m_{2}=\bar{c} m_{1}+i k m_{2}}
\end{gathered}
$$

such that $m_{1}(z, k) \rightarrow 1$ and $m_{2}(z, k) \rightarrow 0\left(\left|m_{2}(z, k)\right|=\left|e_{k}(z) m_{2}(z, k)\right| \rightarrow 0\right)$ as $z \rightarrow \infty$. According to the way in [8], we can obtain the following two lemmas.

\section{Lemma 3.1.}

Under the above conditions, there exist two functions $m_{1}(z, k), m_{2}(z, k)$ satisfying the system of complex equations: 


$$
\begin{aligned}
& {\left[m_{1}(z, k)\right]_{k}+r(k) e_{k}(z) \overline{m_{2}(z, k)}=0,} \\
& {\left[m_{2}(z, k)\right]_{k}+r(k) e_{k}(z) \overline{m_{1}(z, k)}=0}
\end{aligned}
$$

where

$T(k)$

\section{Proof.}

In the following we verify the (3.5). From (3.4), we have

$m_{1}$

$\left[m_{1}\right]_{\bar{k}}$

$$
=-T(k) e_{k}(z) \overline{m_{2}(z, k)}
$$

$e_{-k} m_{2}$

$e_{k} \overline{m_{2}}$

$e_{k}\left[e_{-k} m_{1}\right]_{\bar{k}}=m_{1 \bar{k}}-i(\bar{\zeta}-\bar{z}+\bar{z}) m_{1}$

$=m_{1} \bar{k}-i(\bar{\zeta}-\bar{z}) m_{1}-i \bar{z} m_{1}$,

$m_{2 \bar{k}}=e_{k}\left[e_{-k} m_{2}\right]_{\bar{k}}+i \bar{z} m_{2}$

$$
=-T(k) e_{k}(z) \overline{m_{1}}
$$


In addition, from (3.5) it follows that

$$
\begin{aligned}
& {\left[m_{1}(z, k)+m_{2}(z, k)\right]_{\bar{k}}=-T(k) e_{k}(z)\left[\overline{m_{1}(z, k)}+\overline{m_{2}(z, k)}\right],} \\
& {\left[m_{1}(z, k)-m_{2}(z, k)\right]_{\bar{k}}=T(k) e_{k}(z)\left[\overline{m_{1}(z, k)}-\overline{m_{2}(z, k)}\right] .}
\end{aligned}
$$

It is easy to see that

$$
\begin{aligned}
& w_{1}(z, k)=m_{1}(z, k)+m_{2}(z, k) \\
& w_{2}(z, k)=m_{1}(z, k)-m_{2}(z, k)
\end{aligned}
$$

satisfy the system of complex equations

$$
\psi_{1 \bar{k}}+T(k) e_{k}(z) \overline{w_{1}}=0, \quad w_{2 \bar{k}}-T(k) e_{k}(z) \overline{w_{2}}=0
$$

with the conditions $\psi_{1}=e^{i k z_{1}} \Psi_{1} \cdots 1$ and $\psi_{2}=-i e^{i k z_{1}} \Psi_{2} \cdots 1$ for sufficient large $|k|$, and $\Psi_{1}^{\prime}=e^{-i k z_{\psi_{1}}}, \Psi_{2}^{\prime}=i e^{-i k z_{\psi_{2}}}$ are the solutions of the complex equation

$$
\left[\Psi^{\prime}\right] \bar{k}+T(k) \bar{\Psi}=0 \text { for } k \in \mathrm{C} \text {. }
$$

Later on we will verify $T(k) E L_{\infty}(C)$.

Similarly to the way from (3.2) to (3.6), we can obtain the following result.

Lemma 3.2.

Under the above conditions, there exist two functions $w_{1}(z, k), w_{2}(z, k)$ satisfying the system of complex equations:

$$
\left[W_{1}(z, k)\right]_{\bar{z}}+c(z) \overline{W_{1}(z, k)}=0, \quad\left[W_{2}(z, k)\right]_{\bar{z}}+c(z) \overline{W_{2}(z, k)}=0 \quad \text { in } \mathrm{C} \text {, }
$$

where 
Proof.

Now we verify that (3.12) and (3.13) are true. Denote

$$
\left.n_{1}=\frac{\left[w_{1}(z, k)+w_{2}(z, k)\right]}{2}, \quad n_{2}=e_{k}(z) \frac{\left[w_{2}(z, k)\right.}{-w_{1}(z, k)}\right]
$$

we see that $n_{1}(z, k), n_{2}(z, k)$ satisfy the system of first-order complex equations

$$
\begin{gathered}
{\left[n_{1}\right]_{k}=T(k) n_{2}, \quad\left[n_{2}\right]_{k}-i z n_{2}=\overline{T(k)} n_{1}, \quad e_{k}\left[e_{-k} n_{2}\right]_{k}=\overline{T(k)} n_{1}} \\
{\left[n_{2}\right]_{k}=e_{k}\left[\frac{\overline{W_{2}}-\overline{W_{1}}}{2}\right]_{k}+i z n_{2}=\overline{T(k)} n_{1}+i z n_{2}}
\end{gathered}
$$

such that $n_{1}(z, k) \rightarrow 1$ and $n_{2}(z, k) \rightarrow 0\left(\left|n_{2}(z, k)\right|=\left|e_{k}(z) n_{2}(z, k)\right| \rightarrow 0\right)$ as $k \rightarrow \infty$. Thus we have 
$\left[n_{1}\right]_{\bar{z}}$

$$
=-c(z) e_{k}(z) \overline{n z(z, k)}
$$

$$
\begin{aligned}
e_{-k} n_{2} & \\
e_{k} n_{2} & \\
e_{k}\left[e_{-k} n_{1}\right]_{\bar{z}} & =n_{1 \bar{z}}-i(\bar{k} r-\bar{k}+\bar{k}) n_{1} \\
& =n_{1 \bar{z}}-i(\bar{k} r-\bar{k}) n_{1}-i \bar{k} n_{1}, \\
n_{2 \bar{z}} & =e_{k}\left[e_{-k} n_{2}\right]_{\bar{z}}+i \bar{k} n_{2}
\end{aligned}
$$

In addition, from (3.12) it follows that

$$
\begin{aligned}
& {\left[n_{1}(z, k)+n_{2}(z, k)\right]_{\bar{z}}+c(z) e_{k}(z)\left[\overline{n_{1}(z, k)}+\overline{n_{2}(z, k)}\right]=0,} \\
& {\left[n_{1}(z, k)-n_{2}(z, k)\right]_{\bar{z}}-c(z) e_{k}(z)\left[\overline{n_{1}(z, k)}-\overline{n_{2}(z, k)}\right]=0 .}
\end{aligned}
$$

It is obvious that

$$
\phi_{1}(z, k)=n_{1}(z, k)+n_{2}(z, k), \quad \phi_{2}(z, k)=n_{1}(z, k)-n_{2}(z, k)
$$

satisfy the system of complex equations

$$
\phi_{1 \bar{z}}+c(z) e_{k}(z) \overline{\varphi_{1}}=0, \quad \phi_{2 \bar{z}}-c(z) e_{k}(z) \overline{\varphi_{2}}=0
$$


with the conditions $\phi_{1}=e^{i k z} W_{1} \sim 1$ and $\phi_{2}=-i e^{i k z} W_{2} \sim 1$ for sufficient large $|z|$, and $W_{1}=e^{-i k z_{\phi_{1}}}, W_{2}=i e^{-i k z_{\phi_{2}}}$ are the solutions of the complex equation

$$
W_{\bar{z}}+C(z) \overline{W(z)}=0 \text { for } z \in \mathrm{C}
$$

From (3.6) and Lemma 3.3 below, the functions $H_{1}(z, k)=W_{1}(z, k), H_{2}(z, k)=W_{2}(z, k)$ on $\Gamma$ can be obtained, then

$T(k)$

$$
\begin{aligned}
& =-\frac{1}{4 \pi} \int_{\Gamma} e^{-i \bar{k} \bar{k}}\left(\overline{W_{1}}-i \overline{W_{2}}\right) d \bar{\zeta} \\
& =\frac{i}{4 \pi} \int_{\Gamma} \bar{y} e^{-i \overline{k z}}\left(\overline{W_{1}}-i \overline{W_{2}}\right) d S .
\end{aligned}
$$

Here we use the Green formula

and for $\Gamma_{0}=\{|z|=1\}, \bar{v}=\bar{z}=e^{-i B}=e^{-i \arg z}$, and

$\Gamma_{j}=\left\{\left|z-z_{j}\right|=r_{j}\right\}, \bar{v}=-\overline{\left(z-z_{j}\right)} / r_{j}=-e^{-i \theta}=-e^{-i \arg \left(z-z_{j}\right)}$,

$j=1, \ldots, N, d \bar{z}=-i \bar{v} d S, S=\theta, z \in \Gamma_{0},-d^{\prime} \bar{z}=-d(\bar{z}-\bar{z} \bar{j})=i \bar{v} d S, S=r j \theta, z \in \Gamma_{j}, j=1, \ldots, N$. This

shows that the function $T(k)$ for $k E C$ is known, and then we can solve the solutions $m_{1}, m_{2}$ of equations in (3.5). On the basis of Lemma 3.2, we can obtain the system of complex equations in (3.12) and the coefficient $c(z)=E(z), y(z) e^{T J A-\overline{T J A}}$ of (3.1). This is just the so-called inverse scattering method. We mention that sometimes $w_{1}(z, k), w_{2}(z, k)$ are written as $w_{1}(z), w_{2}(z)$.

\section{Lemma 3.3.}

Under the above conditions, the functions $h_{1}(z), h_{2}(z)$ as stated in (1.12) are the solutions of the system of integral equations 


$$
\begin{aligned}
& \frac{1}{2}\left(1-i S_{k}\right) h_{1}=e^{-i k z}, \quad \frac{1}{2}\left(1-i S_{k}\right) h_{2}=i e^{-i k z}, \\
& S_{k} h_{1}=\frac{1}{n} \int_{\Gamma} \frac{h_{1}(\zeta) e^{i k(\zeta-z)}}{\zeta-z} d \zeta, \quad S_{k} h_{2}=\frac{1}{n} \int_{\Gamma} \frac{h_{2}(\zeta) e^{i k(\zeta-z)}}{\zeta-z} d \zeta .
\end{aligned}
$$

We first prove one lemma (see [7]).

\section{Lemma 3.4.}

The function $g(z, k)=e^{i k z_{h}},(z)\left(\in R_{h}, j=1,2\right)$ is a solution of the integral equations

$$
\begin{gathered}
\left.g(z, k)+T J A g+T e_{k}\right] \overline{A g}=\left\{\begin{array}{l}
1 \\
i
\end{array} \text { in } \bar{D},\right. \\
g(z, k)=\left\{\begin{array}{l}
e^{i k z_{h}(z)} \\
e^{i k z_{h}(z)}
\end{array} \text { on } \Gamma,\right.
\end{gathered}
$$

if and only if it is a solution of the integral equation

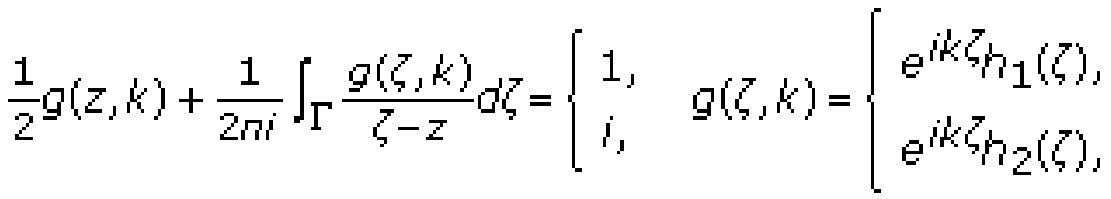

$$
\begin{aligned}
& \frac{h_{1}(z)}{2}+\frac{1}{2 \pi i} \int_{\Gamma} \frac{h_{1}(\zeta) e^{i k(\zeta-z)}}{\zeta-z} d \zeta=e^{-i k z}, \\
& \frac{h_{2}(z)}{2}+\frac{1}{2 n i} \int_{\Gamma} \frac{h_{2}(\zeta) e^{i k(\zeta-z)}}{\zeta-z} d \zeta=i e^{-i k z} \text { on } \Gamma \text {. }
\end{aligned}
$$

\section{Proof.}

It is clear that we can only discuss the case of $h_{1}$. If $g(z, k)$ is a solution of (3.24), then $g \bar{z}=-7 A g-e_{k} \overline{A g}$. On the basis of the Pompeiu formula 


$$
\begin{aligned}
g(z, k) & =\frac{1}{2 n i} \int_{\Gamma} \frac{g(\zeta, k)}{\zeta-z} d \zeta-\tau[g(\zeta, k)] \bar{\zeta} \\
& \left.\left.=\frac{1}{2 n i} \int_{\Gamma} \frac{g(\zeta, k)}{\zeta-z} d \zeta-\tau[] \mathrm{Ag}+e_{k}\right] \overline{\mathrm{Ag}}\right] \text { in } D
\end{aligned}
$$

(see [10, Chapters I and III]), we have

$$
\left.g(z, k)+T 7 A g+T e_{k}\right] \overline{A g}=1=\frac{1}{2 n i} \int_{\Gamma} \frac{g(\zeta, k)}{\zeta-z} d \zeta \text { in } D
$$

where $g(\zeta, k)=e^{i k h_{1}(\zeta)}$ on $\Gamma$. Moreover by using the Plemelj-Sokhotzki formula for Cauchy type integral (see $[12,13])$

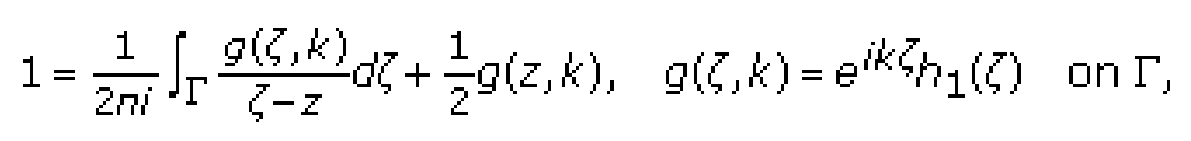

which is the formula (3.25).

On the contrary if (3.25) is true, then there exists a solution of equation $9 \bar{z}=-A g g-e_{k} \overline{A g}$ in $\bar{D}$

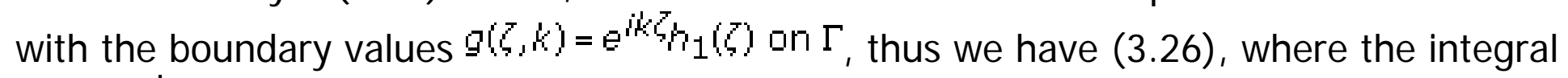
$\left.(1 / 2 \pi i)\right|_{\Gamma}(g(\zeta, k) /(\zeta-z)) \sigma \zeta$ in $\sigma$ is analytic, whose boundary value on $\Gamma$ is

$$
\lim ^{\prime}(E D) \rightarrow z(E \Gamma) \frac{1}{2 n i} \int_{\Gamma} \frac{g(\zeta, k)}{\zeta-z^{\prime}} d \zeta=\frac{1}{2} g(z, k)+\frac{1}{2 n i} \int_{\Gamma} \frac{g(\zeta, k)}{\zeta-z} d \zeta=1
$$

hence

$$
\frac{1}{2 \pi i} \int_{\Gamma} \frac{g(\zeta, k)}{\zeta-z} d \zeta=1 \text { in } D
$$

and the formula (3.24) is true.

Proof of Lemma 3.3.

On the basis of the theory of integral equations (see $[12,13]$ ), we can obtain the solutions 
$h_{1}(z)$ and $h_{2}(z)$ of (3.23). From Lemma 3.4, we define the functions

$$
\begin{array}{r}
W_{1}(z, k)=\left\{\begin{aligned}
& e^{-i k z}-\frac{1}{2 n i} \int_{\Gamma} \frac{t_{1}(\zeta) e^{i k(\zeta-z)}}{\zeta-z} d \zeta, z \in \mathrm{C} \backslash \bar{D}, \\
& z \in \bar{D},
\end{aligned}\right. \\
W_{2}(z, k)=\left\{\begin{aligned}
i e^{-i k z}-\frac{1}{2 n i} \int_{\Gamma} \frac{h_{2}(\zeta) e^{i k(\zeta-z)}}{\zeta-z} d \zeta, & z \in \mathrm{C} \backslash \bar{D}, \\
& z \in \bar{D},
\end{aligned}\right.
\end{array}
$$

which are analytic in $\mathrm{C} \backslash \bar{D}$ with the boundary values $h_{1}(z), h_{2}(z)$ on $\Gamma$ respectively, and satisfy the complex equation (3.1).

Moreover according to [6, 7], we can obtain the following two lemmas.

Lemma 3.5.

Under the above conditions, one has

$$
\left\|e^{i k z} W_{1}(z, k)-1\right\|_{W_{b, 2}^{1}(\mathbf{C}(z))} \leq M_{1}, \quad\left\|-i e^{i k z} W_{2}(z, k)-1\right\|_{W_{p, 2}^{1}}(\mathbf{C}(z)) \leq M_{1}, \text { for } k \dot{k} R,
$$

where $P>2$, the positive constant $M_{1}=M_{1}(k, D, R)$ is only dependent on $k, p$ and $R$, and $R$ is a sufficiently large positive number. Moreover the function $T(k)$ in (3.6) satisfies $T(k) \in L_{\text {co }}(\mathrm{C}(k))$. In particular, $T(k) \in L_{p_{1}, 2}(\mathbf{C}(k))$, where $p_{1}\left(0<p_{1}<\infty\right)$ is a non-negative number.

Proof.

From Lemma 3.1, noting that ${ }^{\phi}(z, k) \rightarrow 1, z \rightarrow \infty, j=1,2$, we have 
$\phi 1(z, k)$

$92(2, k)$

On the basis of the result in [10], we can get

$$
\begin{aligned}
\left\|\phi_{1}(z, k)-1\right\|_{W_{p, 2}^{1}(\mathrm{C}(z))} & \\
& \leq M_{2}\left\|C e_{k}(\zeta) \overline{\varphi_{1}(\zeta, k)}\right\|_{i_{p, 2}(\mathrm{C}(z))} \\
& \leq M_{3}\|C\|_{L_{p, 2}(\mathrm{C}(z))} \\
& \leq M_{1}(k, D, R)
\end{aligned}
$$

in which $|k| \geq R$ and $m_{j}=m_{j}(k, 0, R)(j=2,3)$ are positive constants only dependent on $k, 0$ and R. Similarly, we can obtain the second estimate in (3.32).

In addition, for

$T(k)$

we have

$\|T(k)\|_{i_{\infty}}(\mathrm{C})$

$$
\leq \frac{1}{n}\|C\|_{i_{0}, 2}(\mathrm{C}(z))\left\|m_{1}(\zeta, k)\right\|_{i_{Q}, 2}(\mathrm{C}(z)) \|_{i_{\infty}}(\mathrm{C}(k))
$$

in which $q=p /(D-1), 1<q<2$. It is not difficult to see that $T(k) \in L_{p_{1}, 2}(\mathrm{C}(k))$, where $p_{1}\left(0<D_{1}<\infty\right)$ is a non-negative constant.

Lemma 3.6. 
Under the above conditions, one can find the coefficients $Q=Q(z)$ of the complex system of first-order equations $D_{k} m_{2}=m_{2 z}-i k m_{2}=Q m_{1}(Q=\bar{C})$ in $D$ as follows

\section{$Q(z)$}

in which $d \sigma_{k}=d R e k d I m k$.

Proof.

From the formula (3.4), we can get

$=\pi r^{2} Q(z)$,

where $m_{1}(z, k) \rightarrow 1$ as $k \rightarrow \infty$, hence the the formula (3.37) is true.

Theorem 3.7.

For the inverse problem of Problem DN for (1.3) with Condition $C$, one can reconstruct the coefficients $\bar{\theta}(\zeta)$ and $b(\zeta)$.

Proof.

Similarly to [9], we will use the generalized Cauchy formula

$$
F(z)=\frac{1}{2 n i} \int_{\Gamma} \Omega_{1}(z, \zeta) F d \zeta-\frac{1}{2 n i} \int_{\Gamma} \Omega_{2}(z, \zeta) \bar{F} d \bar{l} \text { in } D
$$

for the complex equation 


$$
F_{\bar{z}}=\left[e^{-T A J}\right]_{\bar{z}}=e^{-T A J^{\prime}}(-A \gamma)=-\bar{C}\left(\frac{y}{\bar{y}}\right) e^{-\overline{T A J}}=-\overline{C F} \frac{\bar{y}}{\bar{y}}
$$

to find the function $F=F(z)=e^{-T A z}$ in $D$, in which $\Omega_{1}(z, \zeta), \Omega_{2}(z, \zeta)$ are the standard kernels of equation (3.40) (see [10, Chapter III]). In fact, denote $F=e^{-T A J}$ in $D$, and $F=e^{-T A J}$ on $\Gamma$ is known from Theorem 2.5, then according to (3.39), we can find the function $F(z)$ in $D$. Moreover from

$$
\left.\left.[-\ln F]_{\bar{z}}=\overline{C F}\right] / F \bar{y}=\bar{C}\left(\frac{\bar{J}}{\bar{y}}\right) e^{T A \bar{\gamma}-\overline{T A J}}=A\right] \text { in } D
$$

thus the coefficient $A=[a(\zeta)+i b(\zeta)] / 4$ in $G$ is obtained.

\section{The Global Uniqueness Result for Inverse Problem of First-Order Elliptic Complex Equations from Dirichlet to Neumann Map}

For the elliptic equation of second-order

$$
u_{j z}+u_{j \eta}+a_{j} u+b_{j} u{ }_{j}=0 \text { in } G, j=1,2
$$

in which $\bar{a}_{j}=\bar{a}_{j}(\zeta), b_{j}=b_{j}(\zeta)$ are real functions of $\zeta=\xi+i n(E G, j=1,2)$, and $\bar{a}_{j}, b_{j} \in L_{D}(\bar{G}), j=1,2, D(2)$ is a positive constant. Moreover define ${ }^{\bar{a}} j=b_{j}=0(j=1,2)$ in $\mathrm{C} \backslash G$. Denote

$$
W_{j}(\zeta)=u_{j}+i V_{j}=\frac{\left[u_{j \zeta}-i u_{j g}\right]}{2}=u_{j \zeta}=\overline{u_{j \zeta}} \text { in } G, j=1,2,
$$

and we can get 


$$
\begin{aligned}
W_{j \bar{\gamma}} & =\frac{\left[W_{j \xi}+i W_{j n}\right]}{2} \\
& =-\frac{1}{4}\left[a_{j} L_{j} \varepsilon+b_{j} L_{j n}\right] \\
& =-A_{j}(\zeta) W_{j}-E_{j}(\zeta) \overline{W_{j}} \\
& =-2 \operatorname{Re}\left[A_{j} \overline{W_{j}}\right] \text { in } G, j=1,2
\end{aligned}
$$

where $A_{j}=A_{j}(\zeta)=\overline{B_{j}(\zeta)}=\left[\bar{a} j+i b_{j}\right] / 4, j=1,2$. As stated in Section 1, suppose that the above equations satisfy Condition $C$, and through a conformal mapping $z=z(\zeta)$, the complex equations in (4.3) can be reduced to the following form

$$
\begin{aligned}
& W_{j \bar{z}}=-\overline{\zeta r(z)}\left\{A_{j}[\zeta(z)] W_{j}+E_{j}[\zeta(z)] \overline{W_{j}}\right\}, \\
& w_{j z}=-2 \operatorname{Re}\left\{A_{j}[\zeta(z)] \overline{\left.\zeta^{\prime}(z) u_{j z}\right\}}\right. \\
& =-2 \operatorname{Re}\left\{A_{j}[\zeta(z)](z) w_{j}\right\} \text { in } D, j=1,2,
\end{aligned}
$$

where $D$ is a circular domain, and $\gamma(z)=\overline{\zeta^{\prime}(z)}$.

If ${ }^{W} j(z)=u_{j z}(j=1,2)$ are the corresponding solutions of (4.4) from the Dirichlet to Neumann maps $A_{j}(j=1,2)$, and $A_{1}=A_{2}=A_{1}$, then the boundary conditions of the inverse boundary value problem for second-order elliptic equations in (4.1) from Dirichlet to Neumann map can be reduced to

$$
w_{j}(z)=u_{j z}=h(z) \text { on } \Gamma, j=1,2
$$

where $h(z)\left(E C_{d}(\Gamma), 0<a \leq(0-2) / p\right)$ is a known complex function. In the following we will prove the uniqueness theorem as follows.

\section{Theorem 4.1.}

For the inverse problem of Problem $D N$ for (1.1) (or (1.3)) with Condition $C$, one can uniquely determine the coefficients $\bar{a}, b$. In other words, if $A_{1}=A_{2}$ for $(4.1)$, then $\bar{a}_{1}=\bar{a}_{2}, b_{1}=b_{2}$.

We first prove the Carleman estimate (see [7]). 
If the complex function $u(z) \in W_{D}^{1}(D)$ with the condition $u(z)=\square$ on $\Gamma$, and the real function $\phi(z) \in W_{D}^{2}(D)(0>2)$ then one has the Carleman estimate

Proof.

It is sufficient to prove the equality

in which $w(z) \in W_{D}^{2}(D)$, and $u(z) \in W_{D}^{1}(0)$ with the condition $u(z)=\square$ on $\Gamma$. We first consider the complex form of the Green formula about $v=u_{z}$

with $u \in C^{2}(\bar{D})$.

If $u(z), q(z)$ are the above functions, by using the Green formula, we have 
thus

This is just the formula (4.7) for $u(z) \in c^{2}(\bar{D})$. Due to the density of $C^{2}(\bar{D})$ in $W_{D}^{1}(D)(0>2)$, it is known that (4.7) is also true for $u(z) \in W_{D}^{1}(D)$ with the condition $u(z)=0$ on $\Gamma$.

\section{Lemma 4.3.}

Under the above conditions, one can derive

$$
C_{1}=C_{2}, \quad z \in D
$$

Proof.

On the basis of $h_{1}(z)=h_{2}(z)$ on $\Gamma$, and the results of Lemmas 3.1 and 3.2, it follows that the corresponding coefficients $T_{1}(k)=T_{2}(k)$, and then $C_{1}(z)=c_{2}(z)$ in $\bar{v}$. This shows that the formula (4.11) is true. 
Proof of Theorem 4.1.

Similarly to [7], we can prove

$$
A_{1}=A_{2} \text { in } D \text {. }
$$

From (4.11), we have

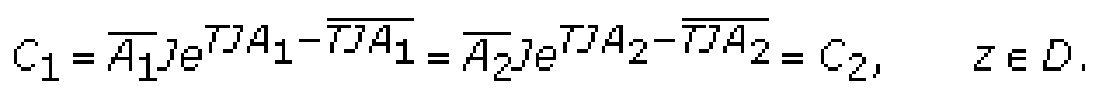

If we define $A_{j}(z)=0, z \in C \backslash D$, then $C_{1}=C_{2}, z \in C$, and denote

$$
E(z)=7\urcorner\left(A_{2}-A_{1}\right), \quad E_{\bar{z}}=7\left(A_{2}-A_{1}\right), \quad F(z)=e^{\overline{T\left(A_{2}-A_{1}\right)}-T \ni\left(A_{2}-A_{1}\right)},
$$

one gets

$$
J A_{1}=F(z) J A_{2}, \quad E(z)=T 7\left(A_{2}-A_{1}\right)=T(1-F(z)) J A_{2} \text { in } \mathrm{C}
$$

Setting that $i \theta(z)=\overline{T 7\left(A_{2}-A_{1}\right)}-T 7\left(A_{2}-A_{1}\right)$, obviously $\theta(z)$ is a real function, and

$$
\begin{aligned}
& |1-F(z)|=\left|e^{i \theta}-1\right|=\left|e^{i \theta / 2}-e^{-i \theta / 2}\right|=2\left|\sin \left(\frac{\theta}{2}\right)\right| \leqslant|\theta| \\
& =\left|\overline{T\left(A_{2}-A_{1}\right)}-7 \eta\left(A_{2}-A_{1}\right)\right| \leq 2\left|T 7\left(A_{2}-A_{1}\right)\right|=2|E(z)| \text {, } \\
& \left.[E(Z)]_{\bar{z}}=\right]\left(A_{2}-A_{1}\right) \text {, } \\
& \left|[E(z)]_{\bar{z}}\right| \leq\left|\jmath A_{2}\right||1-F(z)| \leq 2\left|\jmath A_{2}\right||E(z)| \text {, }
\end{aligned}
$$

where $E(z)=T 7\left(A_{2}-A_{1}\right)=\square$ on $\Gamma$ is derived from Theorem 2.5.

Finally we use the Carleman estimate for $u(z)=E(z)$ and $(4.16)$, and can get 
Taking into account $A_{2} \in L_{D}(D), D>2$, and choosing

it is easy to see that $\Delta \phi=4 \phi_{z} \bar{z}=18|J A|^{2}$ in $D$, and then

Consequently

$$
7\left(A_{2}-A_{1}\right)=0, \quad A_{2}-A_{1}=0 \text { in } D,
$$

this shows the coefficients $\bar{a}_{1}=a_{2}, b_{1}=b_{2}$ of equations in (4.1) in $G$.

\begin{tabular}{|l|l|l|}
\hline \multicolumn{2}{|c|}{ BodyRef } \\
\hline FileRef & $:$ & BodyRef/PDF/13661_2008_Article_838.pdf \\
\hline TargetType & $:$ & OnlinePDF \\
\hline
\end{tabular}

\section{Acknowledgment}

The research was supported by the National Natural Science Foundation of China (10671207). 


\section{References}

1. Kirsch A: An Introduction to the Mathematical Theory of Inverse Problems, Applied Mathematical Sciences. Volume 120. Springer, New York, NY, USA; 1996:x+282.

2. Isakov V: Inverse Problems for Partial Differential Equations, Applied Mathematical Sciences. Volume 127. 2nd edition. Springer, New York, NY, USA; 2006:xiv+344.

3. Sung L-Y: An inverse scattering transform for the Davey-Stewartson II equations-I. Journal of Mathematical Analysis and Applications 1994,183(1):121-154. 10.1006/jmaa.1994.1136

4. Sung L-Y: An inverse scattering transform for the Davey-Stewartson II equations-II. Journal of Mathematical Analysis and Applications 1994,183(2):289-325. 10.1006/jmaa.1994.1145

5. Sung L-Y: An inverse scattering transform for the Davey-Stewartson II equations-III. Journal of Mathematical Analysis and Applications 1994,183(3):477494. 10.1006/jmaa.1994.1155

6. Brown RM, Uhlmann GA: Uniqueness in the inverse conductivity problem for nonsmooth conductivities in two dimensions. Communications in Partial Differential Equations 1997,22(5-6):1009-1027. 10.1080/03605309708821292

7. Cheng $\mathrm{J}$, Yamamoto $\mathrm{M}$ : The global uniqueness for determining two convection coefficients from Dirichlet to Neumann map in two dimensions. Inverse Problems 2000,16(3):L25-L30. 10.1088/0266-5611/16/3/101

8. Tamasan A: On the scattering method for the -equation and reconstruction of convection coefficients. Inverse Problems 2004,20(6):1807-1817. 10.1088/0266$5611 / 20 / 6 / 007$

9. Tong ZL, Cheng J, Yamamoto M: A method for constructing the convection coefficients of elliptic equations from Dirichlet to Neumann map. Science in China. Series A 2004, 34: 752-766.

10. Vekua IN: Generalized Analytic Functions. Pergamon Press, Oxford, UK; 1962.

11. Wen G, Begehr HGW: Boundary Value Problems for Elliptic Equations and Systems, Pitman Monographs and Surveys in Pure and Applied Mathematics. Volume 46. Longman Scientific \& Technical, Harlow, UK; 1990:xii+411.

12. Mushelishvili NI: Singular Integral Equation. P. Noordhoff, Groningen, The Netherlands; 1953.

13. Wen G: Conformal Mappings and Boundary Value Problems, Translations of Mathematical Monographs. Volume 106. American Mathematical Society, Providence, RI, USA; 1992:viii+303. 